ISSN 1561-2430 (Print)

ISSN 2524-2415 (Online)

УДК 519.6

https://doi.org/10.29235/1561-2430-2020-56-1-72-83

Поступила в редакцию 22.11.2019

Received 22.11.2019

\author{
Э. А. Айрян ${ }^{1,2}$, М. Гнатич ${ }^{1,3,4}$, В. Б. Малютин \\ ${ }^{1}$ Объединенный институт ядерных исследований, Дубна, Россия \\ ${ }^{2}$ Российский университет дружбы народов, Москва, Россия \\ ${ }^{3}$ Институт экспериментальной физики Словацкой академии наук, Кошице, Словакия \\ ${ }^{4}$ Университет Павла Йозефа Шафарика, Кошице, Словакия \\ ${ }^{5}$ Институт математики Нацииональной академии наук Беларуси, Минск, Беларусь

\section{ПРИБЛИЖЕННОЕ ВЫЧИСЛЕНИЕ ФУНКЦИОНАЛЬНЫХ ИНТЕГРАЛОВ, ПОРОЖДЕННЫХ РЕЛЯТИВИСТСКИМ ГАМИЛЬТОНИАНОМ}

Аннотация. Рассматривается приближенное вычисление матричнозначных функциональных интегралов специального вида, порожденных релятивистским гамильтонианом. Метод вычисления функциональных интегралов основан на разложении по собственным функциям гамильтониана, порождающего функциональный интеграл. Для нахождения собственных функций и собственных значений исходный гамильтониан рассматривается в виде суммы невозмущенного оператора и малой поправки к нему и используется теория возмущений. Собственные значения и собственные функции невозмущенного оператора вычисляются с помощью метода последовательностей Штурма и метода обратной итерации. Такой подход позволяет значительно уменьшить счетное время и объем используемой памяти компьютера по сравнению с другими известными методами.

Ключевые слова: функциональные интегралы, релятивистский гамильтониан, теория возмущений, собственные функции гамильтониана, последовательность Штурма

Для цитирования. Айрян, Э. А. Приближенное вычисление функциональных интегралов, порожденных релятивистским гамильтонианом / Э. А. Айрян, М. Гнатич, В. Б. Малютин // Вес. Нац. акад. навук Беларусі. Сер. фіз.-мат. навук. - 2020. - Т. 56, № 1. - С. 72-83. https://doi.org/10.29235/1561-2430-2020-56-1-72-83

\author{
Edik A. Ayryan ${ }^{1,2}$, Michal Hnatic ${ }^{1,3,4}$, Victor B. Malyutin ${ }^{5}$ \\ ${ }^{1}$ Joint Institute for Nuclear Research, Dubna, Russia \\ ${ }^{2}$ RUDN University, Moscow, Russia \\ ${ }^{3}$ Slovak Academy of Sciences, Košice, Slovakia \\ ${ }^{4}$ P. J. Šafárik University, Košice, Slovakia \\ ${ }^{5}$ Institute of Mathematics of the National Academy of Sciences of Belarus, Minsk, Belarus
}

\title{
APPROXIMATE EVALUATION OF FUNCTIONAL INTEGRALS GENERATED BY THE RELATIVISTIC HAMILTONIAN
}

\begin{abstract}
An approximate evaluation of matrix-valued functional integrals generated by the relativistic Hamiltonian is considered. The method of evaluation of functional integrals is based on the expansion in the eigenfunctions of Hamiltonian generating the functional integral. To find the eigenfunctions and the eigenvalues the initial Hamiltonian is considered as a sum of the unperturbed operator and a small correction to it, and the perturbation theory is used. The eigenvalues and the eigenfunctions of the unperturbed operator are found using the Sturm sequence method and the reverse iteration method. This approach allows one to significantly reduce the computation time and the used computer memory compared to the other known methods.

Keywords: functional integrals, relativistic Hamiltonian, perturbation theory, eigenfunctions of Hamiltonian, Sturm sequences

For citation. Ayryan E. A., Hnatic M., Malyutin V. B. Approximate evaluation of functional integrals generated by the relativistic Hamiltonian. Vestsi Natsyianal'nai akademii navuk Belarusi. Seryia fizika-matematychnykh navuk = Proceedings of the National Academy of Sciences of Belarus. Physics and Mathematics series, 2020, vol. 56, no. 1, pp. $72-83$ (in Russian). https://doi.org/10.29235/1561-2430-2020-56-1-72-83
\end{abstract}

Введение. Существуют разнообразные методы приближенного вычисления функциональных интегралов. Один из часто используемых - это вычисление интегралов методом Монте-Карло [1-3], который основан на том, что вычисляемый интеграл представляется как математическое ожидание некоторой случайной величины, среднее арифметическое независимых реализаций

(C) Айрян Э. А., Гнатич М., Малютин В. Б., 2020 
которой дает приближенное значение данному интегралу. Существуют методы приближенного вычисления функциональных интегралов, базирующиеся на дискретизации пространства и времени. Часто применяются подходы, не требующие дискретизации и основанные на использовании приближенных формул, являющихся точными на классе функциональных многочленов заданной степени (см. [1, 4-6]). Такие формулы называются формулами заданной степени точности и широко применяются для приближенного вычисления функциональных интегралов. Можно также отметить метод вычисления функциональных интегралов, использующий разложение действия относительно классической траектории [7-10]. В [11-13] был предложен метод вычисления функциональных интегралов, основанный на разложении по собственным функциям нерелятивистского гамильтониана, порождающего функциональный интеграл.

В данной статье рассматривается применение метода, предложенного в [11-13], для приближенного вычисления функциональных интегралов, порожденных релятивистским гамильтонианом. В отличии от [11-13], в настоящей работе дополнительно используется теория возмущений. Для нахождения собственных функций и собственных значений исходный гамильтониан рассматривается в виде суммы невозмущенного оператора и малой поправки к нему. Собственные значения и собственные функции невозмущенного оператора находятся с помощью метода последовательностей Штурма и метода обратной итерации. В разделе 1 вводится функциональный интеграл, порожденный релятивистским гамильтонианом, и предлагается метод для его вычисления. В разделе 2 приводятся результаты численного эксперимента.

1. Аналитические результаты. В данном разделе рассматриваются матричнозначные функциональные интегралы, которые, следуя работам [14, 15], определяются на пространстве функций $x(\tau), s \leq \tau \leq t$, удовлетворяющих условию $x(s)=0$ и условию Липшица с порядком, равным единице, т. е. для любых $s \leq r_{1}<r_{2} \leq t,\left|x\left(r_{2}\right)-x\left(r_{1}\right)\right| \leq M\left|r_{2}-r_{1}\right|$. Интегралы определяются равенством

$$
\begin{gathered}
\int \exp \left\{-\int_{s}^{t} V(x(\tau)) d \tau\right\} d \mu(x)= \\
=\lim _{j} \int_{j}(n) \int_{j} \exp \left\{-\int_{s}^{t} V\left(\sum_{j=1}^{n} x_{j} \chi_{] t_{j-1}, t_{j}\right]}(\cdot)\right) d \tau\right\} \prod_{j=n}^{1} S\left(t_{j}-t_{j-1}, x_{j}-x_{j-1}\right) d x_{1} \ldots d x_{n},
\end{gathered}
$$

если этот предел существует для любого разбиения отрезка $[s, t]$ точками $s=t_{0}<t_{1}<\ldots<t_{n}=t$.

Здесь $x_{j}=x\left(t_{j}\right), \chi_{\left.] t_{j-1}, t_{j}\right]}(\tau)$ - характеристическая функция интервала $\left.] t_{j-1}, t_{j}\right] ; S\left(t_{j}-t_{j-1}, x_{j}-x_{j-1}\right)-$ переходная функция, являющаяся фундаментальным решением уравнения

$$
\frac{\partial S(t, x)}{\partial t}=a \alpha \frac{\partial S(t, x)}{\partial x}+b \beta S(t, x)
$$

где $a, b$ - вещественные параметры, $\alpha, \beta$ - антикоммутирующие матрицы, т. е. $\alpha \beta+\beta \alpha=0$.

Этот интеграл связан с ядром оператора эволюции $[14,15]$, а именно:

$$
\int \exp \left\{-\int_{s}^{t} V(x(\tau)) d \tau\right\} d \mu(x)=\int_{-\infty}^{+\infty} K\left(0, x_{t}\right) d x_{t},
$$

где $K\left(x_{s}, x_{t}\right)$ - ядро оператора $\exp (t H), H=a \alpha \frac{\partial}{\partial x}+b \beta-V(x)$.

Наша цель - использовать для вычисления функционального интеграла разложение функции $K\left(x_{s}, x_{t}\right)$ по собственным функциям гамильтониана $H$.

Для релятивистского гамильтониана $H$ параметры $a, b$ имеют вид $a=-c, b=-m c^{2}$, где $m-$ масса частицы, $c$ - скорость света [16]. Поэтому мы рассмотрим вычисление собственных значений и собственных векторов оператора $H$ при больших значениях $|b|$. Будем также считать, что 


$$
\alpha=\left(\begin{array}{ll}
0 & 1 \\
1 & 0
\end{array}\right), \quad \beta=\left(\begin{array}{cc}
1 & 0 \\
0 & -1
\end{array}\right)
$$

При сделанных предположениях уравнения для собственных значений $E$ и собственных функций $(\phi(x), \psi(x))^{T}(T-$ знак транспонирования) оператора $H$ имеют вид

$$
\begin{aligned}
& a \partial_{x} \psi(x)+b \phi(x)-V(x) \phi(x)=E \phi(x), \\
& a \partial_{x} \phi(x)-b \psi(x)-V(x) \psi(x)=E \psi(x),
\end{aligned}
$$

где $\partial_{x}-$ производная по переменной $x$.

Обозначим $E^{\prime}=E+b$. Тогда

$$
\begin{gathered}
\left(E^{\prime}-2 b+V(x)\right) \phi(x)=a \partial_{x} \psi(x), \\
\left(E^{\prime}+V(x)\right) \psi(x)=a \partial_{x} \phi(x) .
\end{gathered}
$$

Из этих равенств получаем уравнение для $\psi(x)$

$$
\left(E^{\prime}+V(x)\right) \psi(x)=a \partial_{x}\left(E^{\prime}-2 b+V(x)\right)^{-1} a \partial_{x} \psi(x) .
$$

При больших $|b|$ верно приближенное равенство [16]

$$
\left(E^{\prime}-2 b+V(x)\right)^{-1} \approx(-2 b)^{-1}\left(1-\frac{E^{\prime}+V(x)}{-2 b}\right),
$$

с помощью которого уравнение (4) преобразуется к виду

$$
\left(E^{\prime}+V(x)\right) \psi(x)=\frac{a^{2}}{-2 b}\left(\left(1-\frac{E^{\prime}+V(x)}{-2 b}\right) \partial_{x}^{2}+\frac{V^{\prime}(x)}{2 b} \partial_{x}\right) \psi(x) .
$$

Умножая обе части этого равенства на

$$
\left(1-\frac{E^{\prime}+V(x)}{-2 b}\right)^{-1} \approx 1+\frac{E^{\prime}+V(x)}{-2 b},
$$

получим

$$
\begin{gathered}
\left(\frac{a^{2}}{-2 b} \partial_{x}^{2}-\left(1+\frac{E^{\prime}+V(x)}{-2 b}\right)\left(E^{\prime}+V(x)\right)+\frac{a^{2}}{-2 b}\left(1+\frac{E^{\prime}+V(x)}{-2 b}\right) \frac{V^{\prime}(x)}{2 b} \partial_{x}\right) \psi(x) \approx \\
\approx\left(\frac{a^{2}}{-2 b} \partial_{x}^{2}-\left(E^{\prime}+V(x)\right)-\frac{\left(E^{\prime}+V(x)\right)^{2}}{-2 b}+\frac{a^{2}}{-2 b} \frac{V^{\prime}(x)}{2 b} \partial_{x}\right) \psi(x) \approx 0 .
\end{gathered}
$$

Для нахождения собственных значений $E_{0 n}^{\prime}$ и собственных функций $\psi(x)$ рассмотрим невозмущенный оператор

$$
\frac{a^{2}}{-2 b} \partial_{x}^{2}-V(x)
$$

и предположим, что для него известны собственные значения $E_{0 n}^{\prime}$ и собственные функции $\psi_{0 n}(x)$, т. е. известны точные решения уравнения 


$$
\left(\frac{a^{2}}{-2 b} \partial_{x}^{2}-V(x)\right) \psi_{0 n}(x)=E_{0 n}^{\prime} \psi_{0 n}(x) .
$$

Собственные значения $E_{0 n}^{\prime}$ мы будем вычислять приближенно методом последовательностей Штурма [17]. Собственные функции $\psi_{0 n}(x)$ будем вычислять приближенно методом обратной итерации [17]. Тогда собственные значения $E^{\prime}$ и собственные функции $\psi_{n}(x)$ возмущенного оператора с $\varepsilon=\frac{1}{-2 b}$ мы ищем в виде [18]

$$
\begin{gathered}
E_{n}^{\prime}=E_{0 n}^{\prime}+\varepsilon E_{1 n}^{\prime}+\varepsilon^{2} E_{2 n}^{\prime}+\ldots, \\
\psi_{n}(x)=\sum_{j=0}^{\infty} c_{j} \psi_{0 j}(x), \\
c_{j}=c_{0 j}+\varepsilon c_{1 j}+\varepsilon^{2} c_{2 j}+\ldots .
\end{gathered}
$$

Для собственного вектора с номером $n c_{0 j}=1$ при $j=n, c_{0 j}=0$ при $j \neq n$.

Для нахождения поправок первого порядка к собственным значениям и собственным векторам подставим (6) в (5). Получим

$$
\begin{gathered}
\sum_{j=0}\left(E_{0 j}^{\prime}-E_{0 n}^{\prime}-\varepsilon E_{1 n}^{\prime}\right)\left(c_{0 j}+\varepsilon c_{1 j}\right) \psi_{0 j}(x)- \\
-\varepsilon\left(E_{0 n}^{\prime}+V(x)\right)^{2} \sum_{j=0} c_{0 j} \psi_{0 j}(x)-\varepsilon \frac{a^{2}}{-2 b} V^{\prime}(x) \partial_{x} \sum_{j=0} c_{0 j} \psi_{0 j}(x)=0 .
\end{gathered}
$$

Учитывая, что $\sum_{j=0}\left(E_{0 j}^{\prime}-E_{0 n}^{\prime}\right) c_{0 j} \psi_{0 j}(x)=0$, последнее равенство запишется в виде

$$
\begin{gathered}
\sum_{j=0}\left(E_{0 j}^{\prime}-E_{0 n}^{\prime}\right) c_{1 j} \psi_{0 j}(x)-E_{1 n}^{\prime} \psi_{0 n}(x)- \\
-\left(E_{0 n}^{\prime}+V(x)\right)^{2} \psi_{0 n}(x)-\frac{a^{2}}{-2 b} V^{\prime}(x) \partial_{x} \psi_{0 n}(x)=0 .
\end{gathered}
$$

Введем обозначение

$$
V_{j n}=-\int_{-\infty}^{\infty} \psi_{0 j}(x)\left(E_{0 n}^{\prime}+V(x)\right)^{2} \psi_{0 n}(x) d x-\frac{a^{2}}{-2 b} \int_{-\infty}^{\infty} \psi_{0 j}(x) V^{\prime}(x) \partial_{x} \psi_{0 n}(x) d x .
$$

Скалярное произведение векторов $\psi_{i j}(x), \psi_{k l}(x)$ определяется через интеграл $\int_{-\infty}^{\infty} \psi_{i j}(x) \psi_{k l}(x) d x$. Умножая скалярно обе части равенства (7) на вектор $\psi_{0 n}(x)$, получим выражение для $E_{1 n}^{\prime}$ :

$$
E_{1 n}^{\prime}=V_{n n} .
$$

Умножая скалярно обе части равенства (7) на вектор $\psi_{0 j}(x)$, получим выражение для $c_{1 j}, j \neq n$ :

$$
c_{1 j}=\frac{V_{j n}}{E_{0 n}^{\prime}-E_{0 j}^{\prime}} .
$$

При этом $c_{1 n}$ должно быть выбрано так, чтобы функция $\psi_{0 n}(x)+\varepsilon \sum_{j=0} c_{1 j} \psi_{0 j}(x)$ была нормирована с точностью до членов первого порядка включительно. Для этого надо положить [18] $c_{1 n}=0$. 
Таким образом, с поправкой первого порядка собственный вектор дается выражением

$$
\psi_{1 n}(x)=\psi_{0 n}(x)+\varepsilon \sum_{j \neq n} \frac{V_{j n}}{E_{0 n}^{\prime}-E_{0 j}^{\prime}} \psi_{0 j}(x) .
$$

Из (3) следует, что функции $\phi_{i j}(x), i=0,1, j=0,1,2, \ldots$, выражаются через $\psi_{i j}(x)$ по формуле

$$
\phi_{i j}(x)=\frac{a}{-2 b}\left(1-\frac{E_{i j}^{\prime}+V(x)}{-2 b}\right) \partial_{x} \psi_{i j}(x) .
$$

Таким образом, функции $\left(\phi_{i j}(x), \psi_{i j}(x)\right)^{T}$ являются собственными функциями оператора $H$ с собственными значениями $E_{i j}^{\prime}-b$, где $i=0,1-$ порядок малости, $j=0,1,2, \ldots-$ номера собственных значений.

При фиксированном $i$ векторы $\left(\phi_{i j}(x), \psi_{i j}(x)\right)^{T}$, вообще говоря, не ортогональны при различных значениях $j$. Для их ортогонализации можно использовать процесс Грама - Шмидта. При этом ортогональные векторы строятся по правилу

$$
\begin{gathered}
\eta_{i 0}=\left(\phi_{i 0}(x), \psi_{i 0}(x)\right)^{T}, \\
\eta_{i j}=\left(\phi_{i j}(x), \psi_{i j}(x)\right)^{T}-\sum_{k=0}^{j-1} \frac{\left\langle\left(\phi_{i j}(x), \psi_{i j}(x)\right)^{T}, \eta_{i k}\right\rangle}{\left\langle\eta_{i k}, \eta_{i k}\right\rangle} \eta_{i k},
\end{gathered}
$$

где

$$
\left\langle\left(\eta_{1}(x), \eta_{2}(x)\right)^{T},\left(\xi_{1}(x), \xi_{2}(x)\right)^{T}\right\rangle=\int_{-\infty}^{+\infty} \eta_{1}(x) \xi_{1}(x) d x+\int_{-\infty}^{+\infty} \eta_{2}(x) \xi_{2}(x) d x .
$$

Для разложения ядра оператора $\exp (t H)$ по вектору $\eta_{i j}$ можно использовать формулу

$$
K\left(x_{s}, x_{t}\right)=\sum_{j=0}\left\langle\eta_{i j}, \eta_{i j}\right\rangle^{-1} \exp (t H) \eta_{i j}\left(x_{t}\right) \eta_{i j}^{T}\left(x_{s}\right) .
$$

Так как согласно (9) $\eta_{i j}$ можно записать в виде

$$
\eta_{i j}=\sum_{k=0}^{j} c_{k}\left(\phi_{i k}(x), \psi_{i k}(x)\right)^{T},
$$

где $c_{k}$ - некоторые константы, то $\exp (t H) \eta_{i j}\left(x_{t}\right)$ можно записать в виде

$$
\exp (t H) \eta_{i j}\left(x_{t}\right)=\sum_{k=0}^{j} c_{k} \exp \left(t\left(E_{i k}^{\prime}-b\right)\right)\left(\phi_{i k}\left(x_{t}\right), \psi_{i k}\left(x_{t}\right)\right)^{T}
$$

Следовательно,

$$
\begin{gathered}
\int \exp \left\{-\int_{s}^{t} V(x(\tau)) d \tau\right\} d \mu(x)= \\
=\int_{-\infty}^{+\infty} \sum_{j=0}\left\langle\eta_{i j}, \eta_{i j}\right\rangle^{-1} \sum_{k=0}^{j} c_{k} \exp \left(t\left(E_{i k}^{\prime}-b\right)\right)\left(\phi_{i k}\left(x_{t}\right), \psi_{i k}\left(x_{t}\right)\right)^{T} \eta_{i j}^{T}(0) d x_{t} .
\end{gathered}
$$

Таким образом, мы получили формулу для вычисления функционального интеграла с помощью собственных значений $E_{i j}^{\prime}-b$, где $i=0,1$ - порядок малости, $j=0,1,2, \ldots-$ номера собственных значений и собственных функций $\left(\phi_{i j}(x), \psi_{i j}(x)\right)^{T}$ гамильтониана $H$. 
2. Численные результаты. В качестве примера рассмотрим вычисление функционального интеграла (1) в случае, когда переходная функция $S$ определяется уравнением (2),

$$
\alpha=\left(\begin{array}{ll}
0 & 1 \\
1 & 0
\end{array}\right), \quad \beta=\left(\begin{array}{cc}
1 & 0 \\
0 & -1
\end{array}\right), \quad V(x)=\frac{x^{2}}{8} .
$$

Для нулевого порядка малости $(i=0)$ и нулевого собственного значения $(j=0)$ формула $(10)$ имеет вид

$$
\begin{aligned}
& \int \exp \left\{-\int_{s}^{t} V(x(\tau)) d \tau\right\} d \mu(x) \approx \\
& \approx \int_{-\infty}^{+\infty}\left\langle\left(\phi_{00}, \psi_{00}\right),\left(\phi_{00}, \psi_{00}\right)\right\rangle^{-1} \exp \left(t\left(E_{00}^{\prime}-b\right)\right)\left(\phi_{00}\left(x_{t}\right), \psi_{00}\left(x_{t}\right)\right)^{T}\left(\phi_{00}(0), \psi_{00}(0)\right) d x_{t}=
\end{aligned}
$$

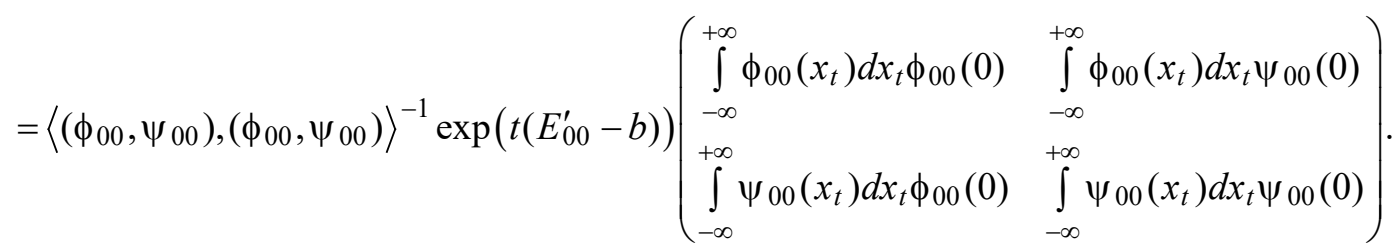

При $s=0, t=2, a=-4, b=-16, m=1, A=5([-A, A]-$ интервал, на котором рассматриваются приближенные собственные функции), $N=100$ ( $N$ - количество интервалов, на которое делится интервал $[-A, A]$ для приближенного вычисления собственных значений и собственных функций)

$$
\begin{gathered}
E_{00}^{\prime} \approx-0,2499, \quad \int_{-\infty}^{+\infty} \psi_{00}\left(x_{t}\right) d x_{t} \approx 2,2366, \quad \int_{-\infty}^{+\infty} \phi_{00}\left(x_{t}\right) d x_{t} \approx 0,00048, \\
\psi_{00}(0) \approx 0,6318, \quad \phi_{00}(0) \approx 0,002, \quad\left\langle\left(\phi_{00}, \psi_{00}\right),\left(\phi_{00}, \psi_{00}\right)\right\rangle^{-1} \approx 0,9923 .
\end{gathered}
$$

Таким образом, для нулевого порядка малости $(i=0)$ и нулевого собственного значения $(j=0)$

$$
\exp (t b) \int \exp \left\{-\int_{s}^{t} V(x(\tau)) d \tau\right\} d \mu(x) \approx\left(\begin{array}{cc}
10^{-6} & 2 \cdot 10^{-4} \\
2 \cdot 10^{-3} & 0,8507
\end{array}\right) .
$$

Для нулевого порядка малости $(i=0)$ и первого собственного значения $(j=0)$ слагаемое с $j=1$ в формуле (10) имеет вид

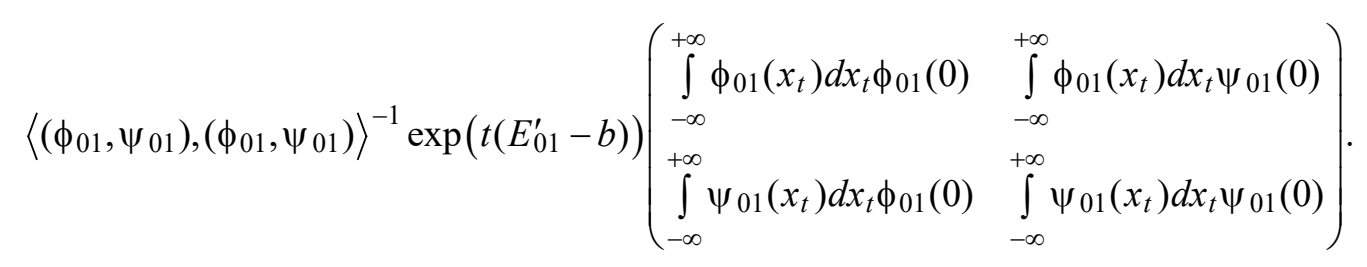

При $s=0, t=2, a=-4, b=-16, m=1, N=100, A=5$

$$
\begin{gathered}
E_{01}^{\prime} \approx-0,7498, \quad \int_{-\infty}^{+\infty} \psi_{01}\left(x_{t}\right) d x_{t} \approx 10^{-6}, \quad \int_{-\infty}^{+\infty} \phi_{01}\left(x_{t}\right) d x_{t} \approx 0,01747, \\
\psi_{01}(0) \approx 8 \cdot 10^{-7}, \quad \phi_{01}(0) \approx 0,08, \quad\left\langle\left(\phi_{01}, \psi_{01}\right),\left(\phi_{01}, \psi_{01}\right)\right\rangle^{-1} \approx 0,9804 .
\end{gathered}
$$

Таким образом, для нулевого порядка малости $(i=0)$ слагаемое с $j=1$ в формуле (10) для $\exp (t b) \int \exp \left\{-\int_{s}^{t} V(x(\tau)) d \tau\right\} d \mu(x)$ дает вклад 


$$
\left(\begin{array}{cc}
2 \cdot 10^{-4} & 2 \cdot 10^{-9} \\
2 \cdot 10^{-8} & 2 \cdot 10^{-13}
\end{array}\right)
$$

Для нулевого порядка малости $(i=0)$ и второго собственного значения $(j=2)$ при $s=0, t=2$, $a=-4, b=-16, m=1, N=100, A=5$

$$
\eta_{02}=\left(\eta_{02,1}, \eta_{02,2}\right) \approx\left(\phi_{02}(x), \psi_{02}(x)\right)^{T}+0,0109\left(\phi_{00}(x), \psi_{00}(x)\right)^{T},\left\langle\eta_{02}, \eta_{02}\right\rangle^{-1} \approx 0,9626
$$

Слагаемое с $j=2$ в формуле (10) имеет вид

$$
\begin{aligned}
& \left\langle\eta_{02}, \eta_{02}\right\rangle^{-1} \exp \left(t\left(E_{02}^{\prime}-b\right)\right)\left(\begin{array}{ll}
\int_{-\infty}^{+\infty} \phi_{02}\left(x_{t}\right) d x_{t} \eta_{02,1}(0) & \int_{-\infty}^{+\infty} \phi_{02}\left(x_{t}\right) d x_{t} \eta_{02,2}(0) \\
\int_{-\infty}^{+\infty} \psi_{02}\left(x_{t}\right) d x_{t} \eta_{02,1}(0) & \int_{-\infty}^{+\infty} \psi_{02}\left(x_{t}\right) d x_{t} \eta_{02,2}(0)
\end{array}\right)+ \\
& +0,0109\left\langle\eta_{02}, \eta_{02}\right\rangle^{-1} \exp \left(t\left(E_{00}^{\prime}-b\right)\right)\left(\begin{array}{ll}
\int_{-\infty}^{+\infty} \phi_{00}\left(x_{t}\right) d x_{t} \eta_{02,1}(0) & \int_{-\infty}^{+\infty} \phi_{00}\left(x_{t}\right) d x_{t} \eta_{02,2}(0) \\
\int_{-\infty}^{+\infty} \psi_{00}\left(x_{t}\right) d x_{t} \eta_{02,1}(0) & \int_{-\infty}^{+\infty} \psi_{00}\left(x_{t}\right) d x_{t} \eta_{02,2}(0)
\end{array}\right) .
\end{aligned}
$$

При указанных параметрах

$$
\begin{aligned}
\int_{-\infty}^{+\infty} \psi_{02}\left(x_{t}\right) d x_{t} \approx 1,5408, & \int_{-\infty}^{+\infty} \phi_{02}\left(x_{t}\right) d x_{t} \approx 9 \cdot 10^{-4}, \quad \psi_{02}(0) \approx-0,4484, \quad \phi_{02}(0) \approx 7 \cdot 10^{-3}, \\
\eta_{02,1}(0) \approx 7 \cdot 10^{-3}, & \eta_{02,2}(0) \approx-0,4421, \quad\left\langle\left(\phi_{02}, \psi_{02}\right),\left(\phi_{02}, \psi_{02}\right)\right\rangle^{-1} \approx 0,9626 .
\end{aligned}
$$

Таким образом, для нулевого порядка малости $(i=0)$ слагаемое с $j=2$ в формуле (10) для $\exp (t b) \int \exp \left\{-\int_{s}^{t} V(x(\tau)) d \tau\right\} d \mu(x)$ дает вклад

$$
\left(\begin{array}{ll}
5 \cdot 10^{-7} & -3 \cdot 10^{-5} \\
9 \cdot 10^{-4} & -0,0596
\end{array}\right)
$$

Для нулевого порядка малости $(i=0)$ и для тех же значений параметров с учетом вклада трех слагаемых получаем

$$
\exp (t b) \int \exp \left\{-\int_{s}^{t} V(x(\tau)) d \tau\right\} d \mu(x) \approx\left(\begin{array}{ll}
2 \cdot 10^{-4} & 2 \cdot 10^{-4} \\
2 \cdot 10^{-3} & 0,7911
\end{array}\right) .
$$

Для первого порядка малости $(i=1)$ и нулевого собственного значения $(j=0)$ формула $(10)$ имеет вид

$$
\begin{aligned}
& \int \exp \left\{-\int_{s}^{t} V(x(\tau)) d \tau\right\} d \mu(x) \approx
\end{aligned}
$$

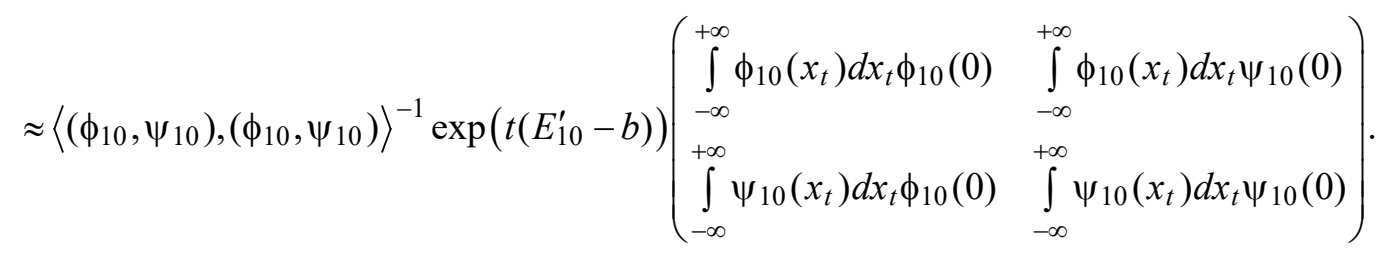


Из формулы (8) следует, что при $s=0, t=2, a=-4, b=-16, m=1, N=100, A=5$

$$
\begin{aligned}
& \psi_{10}(x) \approx \psi_{00}(x)+10^{-4} \psi_{01}(x)+0,0014 \psi_{02}(x), \\
& \phi_{10}(x) \approx \phi_{00}(x)+10^{-4} \phi_{01}(x)+0,0014 \phi_{02}(x) .
\end{aligned}
$$

При указанных параметрах

$$
\begin{gathered}
E_{10}^{\prime} \approx-0,2494, \quad \int_{-\infty}^{+\infty} \psi_{10}\left(x_{t}\right) d x_{t} \approx 2,2388, \quad \int_{-\infty}^{+\infty} \phi_{10}\left(x_{t}\right) d x_{t} \approx 0,00048, \\
\psi_{10}(0) \approx 0,6312, \quad \phi_{10}(0) \approx 0,002, \quad\left\langle\left(\phi_{10}, \psi_{10}\right),\left(\phi_{10}, \psi_{10}\right)\right\rangle^{-1} \approx 0,9923 .
\end{gathered}
$$

Таким образом, для первого порядка малости $(i=1)$ и нулевого собственного значения $(j=0)$

$$
\exp (t b) \int \exp \left\{-\int_{s}^{t} V(x(\tau)) d \tau\right\} d \mu(x) \approx\left(\begin{array}{ll}
6 \cdot 10^{-7} & 2 \cdot 10^{-4} \\
2 \cdot 10^{-3} & 0,8516
\end{array}\right) .
$$

Для первого порядка малости $(i=1)$ и первого собственного значения $(j=1)$ при указанных параметрах из формулы (8), выражающей $\psi_{11}(x)$ через $\psi_{0 j}(x), j=0,1,2$, получаем

$$
\begin{aligned}
& \psi_{11}(x) \approx \psi_{01}(x)+3 \cdot 10^{-4} \psi_{00}(x)-7 \cdot 10^{-6} \psi_{02}(x), \\
& \phi_{11}(x) \approx \phi_{01}(x)+3 \cdot 10^{-4} \phi_{00}(x)-7 \cdot 10^{-6} \phi_{02}(x) .
\end{aligned}
$$

После ортогонализации получим

$$
\eta_{11}=\left(\eta_{11,1}, \eta_{11,2}\right) \approx\left(\phi_{11}(x), \psi_{11}(x)\right)^{T}-4 \cdot 10^{-4}\left(\phi_{10}(x), \psi_{10}(x)\right)^{T},\left\langle\eta_{11}, \eta_{11}\right\rangle^{-1} \approx 0,9804 .
$$

Слагаемое с $j=1$ в формуле (10) имеет вид

$$
\begin{aligned}
& \left\langle\eta_{11}, \eta_{11}\right\rangle^{-1} \exp \left(t\left(E_{11}^{\prime}-b\right)\right)\left(\begin{array}{ll}
\int_{-\infty}^{+\infty} \phi_{11}\left(x_{t}\right) d x_{t} \eta_{11,1}(0) & \int_{-\infty}^{+\infty} \phi_{11}\left(x_{t}\right) d x_{t} \eta_{11,2}(0) \\
\int_{-\infty}^{+\infty} \psi_{11}\left(x_{t}\right) d x_{t} \eta_{11,1}(0) & \int_{-\infty}^{+\infty} \psi_{11}\left(x_{t}\right) d x_{t} \eta_{11,2}(0)
\end{array}\right)- \\
& -4 \cdot 10^{-4}\left\langle\eta_{11}, \eta_{11}\right\rangle^{-1} \exp \left(t\left(E_{10}^{\prime}-b\right)\right)\left(\begin{array}{ll}
\int_{-\infty}^{+\infty} \phi_{10}\left(x_{t}\right) d x_{t} \eta_{11,1}(0) & \int_{-\infty}^{+\infty} \phi_{10}\left(x_{t}\right) d x_{t} \eta_{11,2}(0) \\
\int_{-\infty}^{+\infty} \psi_{10}\left(x_{t}\right) d x_{t} \eta_{11,1}(0) & \int_{-\infty}^{+\infty} \psi_{10}\left(x_{t}\right) d x_{t} \eta_{11,2}(0)
\end{array}\right) .
\end{aligned}
$$

При указанных параметрах

$$
\begin{gathered}
E_{11}^{\prime} \approx-0,7552, \quad \int_{-\infty}^{+\infty} \psi_{11}\left(x_{t}\right) d x_{t} \approx 6 \cdot 10^{-4}, \quad \int_{-\infty}^{+\infty} \phi_{11}\left(x_{t}\right) d x_{t} \approx 0,01747, \\
\psi_{11}(0) \approx 2 \cdot 10^{-4}, \quad \phi_{11}(0) \approx 8 \cdot 10^{-2}, \quad \eta_{11,1}(0) \approx 8 \cdot 10^{-2}, \quad \eta_{11,2}(0) \approx-5 \cdot 10^{-5} .
\end{gathered}
$$

Таким образом, для первого порядка малости $(i=1)$ слагаемое с $j=1$ в формуле (10) для $\exp (t b) \int \exp \left\{-\int_{s}^{t} V(x(\tau)) d \tau\right\} d \mu(x)$ дает вклад 


$$
\left(\begin{array}{cc}
3 \cdot 10^{-4} & -2 \cdot 10^{-7} \\
-3 \cdot 10^{-5} & -3 \cdot 10^{-8}
\end{array}\right)
$$

Для первого порядка малости $(i=1)$ и второго собственного значения $(j=2)$ при указанных параметрах из формулы (8), выражающей $\psi_{12}(x)$ через $\psi_{0 j}(x), j=0,1,2$, получаем

$$
\begin{gathered}
\psi_{12}(x) \approx \psi_{02}(x)-0,0069 \psi_{00}(x)+0,0179 \psi_{01}(x), \\
\phi_{12}(x) \approx \phi_{02}(x)-0,0069 \phi_{00}(x)+0,0179 \phi_{01}(x) .
\end{gathered}
$$

После ортогонализации получим

$$
\begin{gathered}
\eta_{12}=\left(\eta_{12,1}, \eta_{12,2}\right) \approx\left(\phi_{12}(x), \psi_{12}(x)\right)^{T}+0,016\left(\phi_{10}(x), \psi_{10}(x)\right)^{T}-0,018\left(\phi_{11}(x), \psi_{11}(x)\right)^{T}, \\
\left\langle\eta_{12}, \eta_{12}\right\rangle^{-1} \approx 0,9626 .
\end{gathered}
$$

Слагаемое с $j=2$ в формуле (10) имеет вид

$$
\begin{aligned}
& \left\langle\eta_{12}, \eta_{12}\right\rangle^{-1} \exp \left(t\left(E_{12}^{\prime}-b\right)\right)\left(\begin{array}{ll}
\int_{-\infty}^{+\infty} \phi_{12}\left(x_{t}\right) d x_{t} \eta_{12,1}(0) & \int_{-\infty}^{+\infty} \phi_{12}\left(x_{t}\right) d x_{t} \eta_{12,2}(0) \\
\int_{-\infty}^{+\infty} \psi_{12}\left(x_{t}\right) d x_{t} \eta_{12,1}(0) & \int_{-\infty}^{+\infty} \psi_{12}\left(x_{t}\right) d x_{t} \eta_{12,2}(0)
\end{array}\right)+ \\
& +0,016\left\langle\eta_{12}, \eta_{12}\right\rangle^{-1} \exp \left(t\left(E_{10}^{\prime}-b\right)\right)\left(\begin{array}{ll}
\int_{-\infty}^{+\infty} \phi_{10}\left(x_{t}\right) d x_{t} \eta_{12,1}(0) & \int_{-\infty}^{+\infty} \phi_{10}\left(x_{t}\right) d x_{t} \eta_{12,2}(0) \\
\int_{-\infty}^{+\infty} \psi_{10}\left(x_{t}\right) d x_{t} \eta_{12,1}(0) & \int_{-\infty}^{+\infty} \psi_{10}\left(x_{t}\right) d x_{t} \eta_{12,2}(0)
\end{array}\right)- \\
& -0,018\left\langle\eta_{12}, \eta_{12}\right\rangle^{-1} \exp \left(t\left(E_{11}^{\prime}-b\right)\right)\left(\begin{array}{ll}
\int_{-\infty}^{+\infty} \phi_{11}\left(x_{t}\right) d x_{t} \eta_{12,1}(0) & \int_{-\infty}^{+\infty} \phi_{11}\left(x_{t}\right) d x_{t} \eta_{12,2}(0) \\
\int_{-\infty}^{+\infty} \psi_{11}\left(x_{t}\right) d x_{t} \eta_{12,1}(0) & \int_{-\infty}^{+\infty} \psi_{11}\left(x_{t}\right) d x_{t} \eta_{12,2}(0)
\end{array}\right) .
\end{aligned}
$$

При указанных параметрах

$$
\begin{gathered}
E_{12}^{\prime} \approx-1,2677, \quad \int_{-\infty}^{+\infty} \psi_{12}\left(x_{t}\right) d x_{t} \approx 1,5254, \quad \int_{-\infty}^{+\infty} \phi_{12}\left(x_{t}\right) d x_{t} \approx 10^{-3}, \quad \psi_{12}(0) \approx-0,4528, \\
\phi_{12}(0) \approx 8 \cdot 10^{-3}, \quad \eta_{12,1}(0) \approx 7 \cdot 10^{-3}, \quad \eta_{12,2}(0) \approx-0,4427 .
\end{gathered}
$$

Таким образом, для первого порядка малости $(i=1)$ слагаемое с $j=2$ в формуле $(10)$ для $\exp (t b) \int \exp \left\{-\int_{s}^{t} V(x(\tau)) d \tau\right\} d \mu(x)$ дает вклад

$$
\left(\begin{array}{cc}
-2 \cdot 10^{-7} & -2 \cdot 10^{-6} \\
10^{-3} & -0,0607
\end{array}\right)
$$

Для первого порядка малости $(i=1)$ и для тех же значений параметров с учетом вклада трех слагаемых получаем

$$
\exp (t b) \int \exp \left\{-\int_{s}^{t} V(x(\tau)) d \tau\right\} d \mu(x) \approx\left(\begin{array}{ll}
2 \cdot 10^{-4} & 2 \cdot 10^{-4} \\
3 \cdot 10^{-3} & 0,7909
\end{array}\right) .
$$


Для элементов матрицы $\exp (t b) \int \exp \left\{-\int_{s}^{t} V(x(\tau)) d \tau\right\} d \mu(x)$ с индексами $(1,2)$ и $(2,1)$ при $V(x)=\frac{x^{2}}{8}$ известны точные значения, и они равны нулю [19]. Для сравнения на рис. 1, 2 приведены приближенные значения элементов матричнозначного интеграла для различных значений $N$.

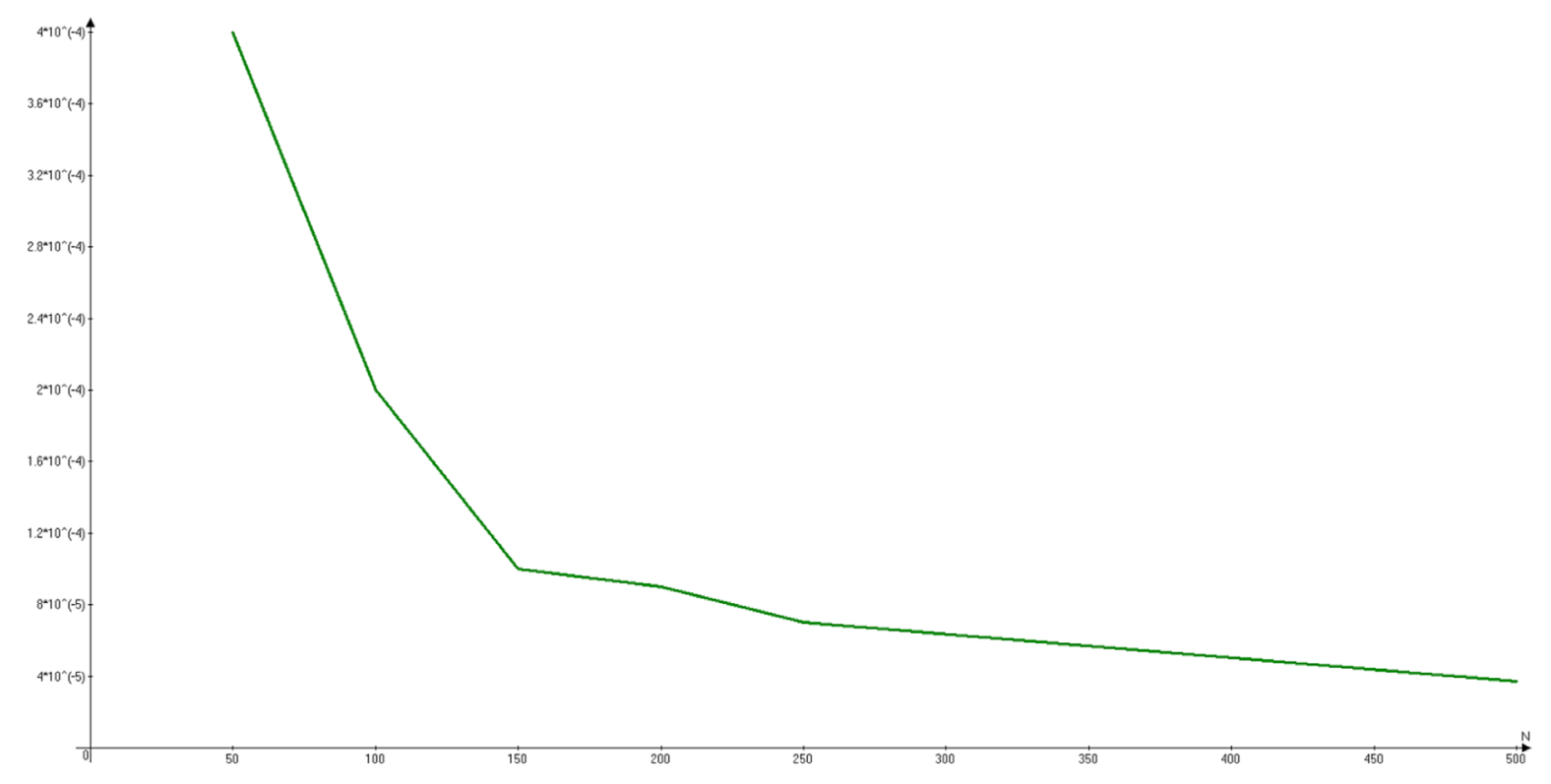

Рис. 1. Приближенные значения элемента матричнозначного интеграла с индексом $(1,2)$ : $s=0, t=2, a=-4, b=-16, m=1, A=5, i=0, j=0$

Fig. 1. Approximate values of the element of the matrix-valued integral with index $(1,2)$ : $s=0, t=2, a=-4, b=-16, m=1, A=5, i=0, j=0$

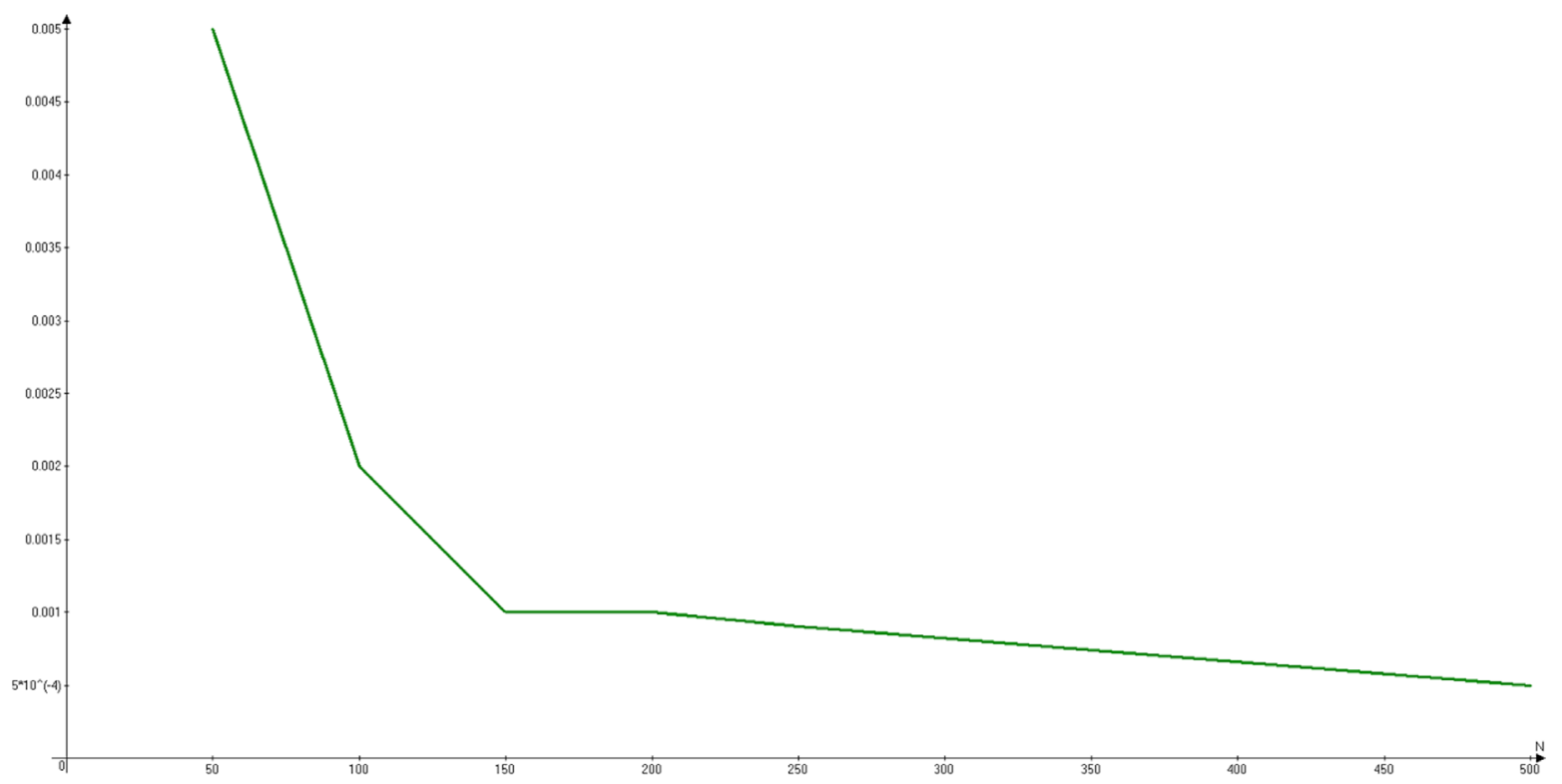

Рис. 2. Приближенные значения элемента матричнозначного интеграла с индексом $(2,1)$ : $s=0, t=2, a=-4, b=-16, m=1, A=5, i=0, j=0$

Fig. 2. Approximate values of the element of the matrix-valued integral with index $(2,1)$ : $s=0, t=2, a=-4, b=-16, m=1, A=5, i=0, j=0$ 
Из рис. 1, 2 видно, что приближенные значения элементов матричнозначного интеграла стремятся к точному значению при увеличении количества интервалов $N$, на которое делится интервал $[-A, A]$ для приближенного вычисления собственных значений и собственных функций.

Таким образом, предложен метод вычисления матричнозначных функциональных интегралов, порожденных релятивистским гамильтонианом, приведены результаты численной апробации метода, основанного на разложении по собственным функциям гамильтониана, порождающего функциональный интеграл. Для нахождения собственных функций и собственных значений исходный гамильтониан рассматривается в виде суммы невозмущенного оператора и малой поправки к нему. Собственные значения и собственные функции невозмущенного оператора находятся с помощью метода последовательностей Штурма и метода обратной итерации.

\section{Список использованных источников}

1. Янович, Л. А. Приближенное вычисление континуальных интегралов по гауссовым мерам / Л. А. Янович. Минск: Наука и техника, 1976. - 382 с.

2. Решение краевых задач методом Монте-Карло / Б. С. Елепов [и др.]. - Новосибирск: Наука, 1980.

3. Сабельфельд, К. К. О приближенном вычислении винеровских континуальных интегралов методом МонтеКарло / К. К. Сабельфельд // Журн. вычисл. математики и мат. физики. - 1979. - Т. 19, № 1. - С. $29-43$.

4. Егоров, А. Д. Приближенные методы вычисления континуальных интегралов / А. Д. Егоров, П. И. Соболевский, Л. А. Янович. - Минск: Наука и техника, 1985. - 309 с.

5. Egorov, A. D. Functional Integrals: Approximate Evaluation and Applications / A. D. Egorov, P. I. Sobolevsky, L. A. Yanovich. - Dordrecht: Kluwer Academic Pabl., 1993. - 400 p. https://doi.org/10.1007/978-94-011-1761-6

6. Егоров, А. Д. Введение в теорию и приложения функционального интегрирования / А. Д. Егоров, Е. П. Жидков, Ю. Ю. Лобанов. - М.: Физматлит, 2006. - 400 c.

7. Feynman, R. P. Quantum mechanics and path integrals / R. P. Feynman, A. R. Hibbs. - New York: McGraw-Hill, 1965. $-382 \mathrm{p}$.

8. Horacio, S. W. Path Integrals for Stochastic Processes: an introduction / S. Wio Horacio. - World Scientific Publ. Company, 2013. - 176 p. https://doi.org/10.1142/8695

9. Применение функциональных интегралов к стохастическим уравнениям / Э. А. Айрян [и др.] // Мат. моделирование. - 2016. - Т. 28, № 11. - С. 113-125.

10. Метод функциональных интегралов для систем стохастических дифференциальных уравнений / Э. А. Айрян [и др.] // Вес. Нац. акад. навук Беларусі. Сер. фіз.-мат. навук. - 2018. - Т. 54, № 3. - С. 279-289. https:// doi. org/10.29235/1561-2430-2018-54-3-279-289

11. Малютин, В. Б. Вычисление функциональных интегралов с помощью последовательностей Штурма / В. Б. Малютин // Вес. Нац. акад. навук Беларусі. Сер. фіз.-мат. навук. - 2016. - № 4. - С. 32-37.

12. Малютин, В. Б. О вычислении функциональных интегралов, порожденных некоторыми нерелятивистскими гамильтонианами / В. Б. Малютин // Вес. Нац. акад. навук Беларусі. Сер. фіз.-мат. навук. - 2018. - Т. 54, № 1. - С. 44-49.

13. Малютин, В. Б. Приближенное вычисление функциональных интегралов, содержащих центробежный потенциал / В. Б. Малютин // Вес. Нац. акад. навук Беларусі. Сер. фіз.-мат. навук. - 2019. - Т. 55, № 2. - С. $152-157$. https://doi.org/10.29235/1561-2430-2019-55-2-152-157

14. Ichinose, T. Propagation of a Dirac particle. A path integral approach / T. Ichinose, H. Tamura // J. Math. Phys. 1984. - Vol. 25, № 6. - P. 1810-1819. https://doi.org/10.1063/1.526360

15. Ichinose, T. The zitterbewegung of a Dirac particle in two-dimensional space-time / T. Ichinose, H. Tamura // J. Math. Phys. - 1988. - Vol. 29, № 1. - P. 103-109. https://doi.org/10.1063/1.528162

16. Шифф, Л. Квантовая механика / Л. Шифф. - М.: Изд-во иностр. лит., 1959. - 473 с.

17. Wilkinson, J. H. The Algebraic Eigenvalue Problem / J. H. Wilkinson. - Oxford, 1965. - 662 p.

18. Ландау, Л. Д. Курс теоретической физики / Л. Д. Ландау, Е. М. Лифшиц. - М.: Наука, 1989. - Т. 3. Квантовая механика. Нерелятивистская теория. -768 с.

19. Ayryan, E. A. Application of functional polynomials to approximation of matrix-valued functional integrals / E. A. Ayryan, V. B. Malyutin // Bulletin of Peoples' Friendship University of Russia. Series Mathematics Informatics Physics. - 2014. - № 1. - P. 55-58.

\section{References}

1. Yanovich L. A. Approximate Evaluation of Continual Integrals with Respect to Gaussian Measures. Minsk, Nauka i tekhnika Publ., 1976. 382 p. (in Russian).

2. Elepov B. S., Kronberg A. A., Mikhailov G. A., Sabelfeld K. K. Solution of Boundary Value Problems by Monte-Carlo Method. Novosibirsk, Science Publ., 1980. 174 p. (in Russian).

3. Sabelfeld K. K. Approximate evaluation of Wiener continual integrals by Monte-Carlo method. Zhurnal vychislitel'noi matematiki i matematicheskoi fiziki = Computational Mathematics and Mathematical Physics, 1979, vol. 19, no. 1, pp. 29-43 (in Russian). 
4. Egorov A. D., Sobolevsky P. I., Yanovich L. A. Approximate Methods of Evaluation of Continual Integrals. Minsk, Nauka i tekhnika Publ., 1985. 309 p. (in Russian).

5. Egorov A. D., Sobolevsky P. I., Yanovich L. A. Functional Integrals: Approximate Evaluation and Applications. Dordrecht, Kluwer Academic Pabl., 1993. 400 p. https://doi.org/10.1007/978-94-011-1761-6

6. Egorov A. D., Zhidkov E. P., Lobanov Yu. Yu. Introduction to Theory and Applications of Functional Integration. Moscow, Fizmatlit Publ., 2006. 400 p. (in Russian).

7. Feynman R. P., Hibbs A. R. Quantum Mechanics and Path Integrals. New York, McGraw-Hill, 1965. 382 p.

8. Horacio S. Wio. Path Integrals for Stochastic Processes: an introduction. World Scientific Publ. Company, 2013.176 p. https://doi.org/10.1142/8695

9. Ayryan E. A., Egorov A. D., Kulyabov D. S., Malyutin V. B., Sevastyanov L. A. Application of functional integrals to stochastic equations. Mathematical Models and Computer Simulations, 2017, vol. 9, no. 3, pp. 339-348. https://doi.org/10.1134/ s2070048217030024

10. Ayryan E. A., Egorov A. D., Kulyabov D. S., Malyutin V. B., Sevastyanov L. A. Functional integrals method for systems of stochastic differential equations. Vestsi Natsyianal'nai akademii navuk Belarusi. Seryia fizika-matematychnykh navuk $=$ Proceedings of the National Academy of Sciences of Belarus. Physics and Mathematics series, 2018, vol. 54, no. 3, pp. 279-289 (in Russian). https://doi.org/10.29235/1561-2430-2018-54-3-279-289

11. Malyutin V. B. Evaluation of functional integrals using Sturm sequences. Vestsi Natsyianal'nai akademii navuk Belarusi. Seryia fizika-matematychnykh navuk = Proceedings of the National Academy of Sciences of Belarus. Physics and Mathematics series, 2016, no. 4, pp. 32-37 (in Russian).

12. Malyutin V. B. Evaluation of functional integrals generated by some nonrelativistic Hamiltonians. Vestsi Natsyianal'nai akademii navuk Belarusi. Seryia fizika-matematychnykh navuk = Proceedings of the National Academy of Sciences of Belarus. Physics and Mathematics series, 2018, vol. 54, no. 1, pp. 44-49 (in Russian).

13. Malyutin V. B. Approximate evaluation of functional integrals with centrifugal potential. Vestsi Natsyianal'nai akademii navuk Belarusi. Seryia fizika-matematychnyk navuk = Proceedings of the National Academy of Sciences of Belarus. Physics and Mathematics series, 2019, vol. 55, no. 2, pp. 152-157 (in Russian). https://doi.org/10.29235/1561-24302019-55-2-152-157

14. Ichinose T., Tamura H. Propagation of a Dirac particle. A path integral approach. Journal of Mathematics and Physics, 1984, vol. 25, no. 6, pp. 1810-1819. https://doi.org/10.1063/1.526360

15. Ichinose T., Tamura H. The zitterbewegung of a Dirac particle in two-dimensional space-time. Journal of Mathematics and Physics, 1988, vol. 29, no. 1, pp. 103-109. https://doi.org/10.1063/1.528162

16. Schiff L. Quantum Mechanics. New York, McCraw-Hill Book Company, 1955. 417 p.

17. Wilkinson J. H. The Algebraic Eigenvalue Problem. Oxford, 1965. 662 p.

18. Landau L. D., Lifshits E. M. Course of Theoretical Physics. Volume 3. Quantum Mechanics. Nonrelativistic Theory. Moscow, Nauka Publ., 1989. 768 p. (in Russian).

19. Ayryan E. A., Malyutin V. B. Application of functional polynomials to approximation of matrix-valued functional integrals. Bulletin of Peoples' Friendship University of Russia. Series Mathematics Informatics Physics, 2014, no. 1, pp. 55-58.

\section{Информация об авторах}

Айрян Эдик Арташевич - кандидат физико-математических наук, заведующий сектором, Лаборатория информационных технологий, Объединенный институт ядерных исследований (ул. Жолио-Кюри, 6, 141980 , г. Дубна, Российская Федерация); Российский университет дружбы народов (РУДН) (ул. Миклухо-Маклая, 6, 117198, г. Москва, Российская Федерация). E-mail: ayrjan@jinr.ru

Михал Гнатич - доктор физико-математических наук наук, заместитель директора, Лаборатория теоретической физики им. Н. Н. Боголюбова, Объединенный институт ядерных исследований (ул. Жолио-Кюри, 6, 141980 , г. Дубна, Российская Федерация); Институт экспериментальной физики Словацкой академии наук (ул. Ватсонова, 47, 04001, г. Кошице, Словацкая Республика); Факультет естествознания, Университет Павла Йозефа Шафарика (Парк Ангелинум, 9, 04001, г. Кошице, Словацкая Республика). E-mail: hnatic@saske.sk

Малютин Виктор Борисович - доктор физикоматематических наук, главный научный сотрудник, Институт математики Национальной академии наук Беларуси (ул. Сурганова, 11, 220072, г. Минск, Республика Беларусь). E-mail: malyutin@im.bas-net.by

\section{Information about the authors}

Edik A. Ayryan - Ph. D. (Physics and Mathematics), Head of Sector, Laboratory of Information Technologies, Joint Institute for Nuclear Research (6, Joliot-Curie Str., 141980, Dubna, Russian Federation); RUDN University (6, MiklukhoMaklay Str., 117198, Moscow, Russian Federation). E-mail: ayrjan@jinr.ru

Michal Hnatic - Dr. Sc. (Physics and Mathematics), Deputy Director, Bogoliubov Laboratory of Theoretical Physics, Joint Institute for Nuclear Research (6, JoliotCurie Str., 141980, Dubna, Russian Federation); Institute of Experimental Physics of the Slovak Academy of Sciences (IEP SAS) (47, Watsonova Str., 04001, Košice, Slovak Republic); Faculty of Science P. J. Safarik University (9, Park Angelinum, 04001, Košice, Slovak Republic). E-mail: hnatic@ saske.sk

Victor B. Malyutin - Dr. Sc. (Physics and Mathematics), Principal Researcher, Institute of Mathematics of the National Academy of Sciences of Belarus (11, Surganov Str., 220072, Minsk, Republic of Belarus). E-mail: malyutin@ im.bas-net.by 